\title{
Generalised Inner Derivations in Semi Prime Rings
}

\author{
Dr. K. L Kaushik \\ Associate Professor,(Head) Department of Mathematics \\ Aggarwal College, Ballabgarh, Faridabad, India
}

\begin{abstract}
Let $A$ be any ring and $f(x y)=f(x) y+x h_{a}(y)$, where $f$ be any generalised inner derivation(G.I.D ) $a$ be the fixed element of $A$.
\end{abstract}

In this paper, it is shown that (i) $h_{a}$ must necessarily be a derivation for semi prime ring $A$. (ii) $\exists$ no generalized inner derivations $f: A \rightarrow A$ such that

or

$$
f(x \circ y)=x \circ y
$$

$$
f(x \circ y)+x \circ y=0 \forall x, y \in A,
$$

We have proved Havala [2] def. p.1147, Herstein [3] Lemma 3.1 p. 1106 as corollaries, along with other results.

\section{INTRODUCTION}

We have defined the G.I.D. of a ring. "Let $A$ be any ring. An additive mapping $f: A \rightarrow A$ is said to be G.I.D if $f(x y)=f(x) y+x h_{a}(y)$

where $h_{a}: A \rightarrow A y \rightarrow[a, y] \forall x, y \in A$, fixed element $a$ $\in A$," Let $G_{I} D(A)$ be the set of all G.I.D of $A$ into itself. We prove that $f(x y z)=f(x) y z+x h_{a}(y z), \forall, x, y, z \in A, f \in G_{I} D(A)$.

In Theorem 2.2, we have shown that $h_{a}$ must necessarily be a derivation on $A$ where $A$ be any semi prime ring. In Corollary 2.3, replacing $h_{a}$ by $d$, we get Havala [2] def. p-1147 of Generalized derivation. In Corollary 2.5 replacing $f$ by $d$, we get Herstein [3] Lemma 3.1, P-1093.

Keywords and phrases. G.I.D, semi prime ring, 2 torsion free semi prime ring, Unity.

A. In Theorem 3.2, we have proved that $\exists$ no G.I.D $f: A \rightarrow$ A such that

or

$$
f(x \circ y) \quad=\quad x \circ y
$$

$$
f(x \circ y)+x \circ y \quad=\quad 0 \forall x, y \in A
$$

and $x \circ y=x y+y x$. For any 2 torsion free semi prime ring A with identity .

Finally in Theorem 4.1 we have proved that $(1+f(1))[x, y]+[a,[x, y]]=0$ for any non-zero Ideal $K$ of $A$ with unity.

\section{Generalized Inner Derivation}

In this section, we study the G.I.D in a ring.

Definition 1.1. (Generalized Inner Derivation): An additive mapping $f: A \rightarrow A$ is said to be G.I.D if $f(x y)=$ $f(x) y+x[a, y]$, for fixed element $a \in A$ and $\forall x, y \in A$

We are taking the definition as

$f(x y)=f(x) y+x h_{a}(y)$

where

$h_{a}: A \rightarrow A y \rightarrow[a, y]$ is the inner derivation.

Let $G_{I} D(A)$ be the set of all G.I.D of $A$ into itself.

Lemma 1.2 If $f \in G_{I} D(A)$. Then $f(x y z)=f(x) y z+x h_{a}(y z), \forall$ $x, y, z \in A$.

Proof. Now

$f(x y z)$

$$
\begin{aligned}
& =f(x y) z+x y[a, z], \\
& =\quad(f(x) y+x[a, y]) z+x y[a, z] \\
& =f(x) y z+x[a, y] z+x y[a, z] \\
& =f(x) y z+x(a y-y a) z+x y(a z- \\
& =\quad z a) \\
& =\quad f(x) y z+x a y z-x y a z+x y a z- \\
& =f(x) y z+x(a y z-y z a) \\
& =f(x) y z+x[a, y z]
\end{aligned}
$$$$
f(x y z) \quad=f(x) y z+x h_{a}(y z)
$$

Hence proved.

$B$. In this section, we take $A$ be any semi prime ring.

Definition 2.1. (Semi prime ring) : Let $A$ be any ring. Then $A$ is said to be semi-prime ring if $x a x=0 \forall a \in A \Rightarrow x$ $=0$

Now $A=$ semi prime ring has the following property: "If $A a=0, a \in A$ then $a=0$ "

This result is used in Theorem 2.2.

Theorem 2.2 Let $A$ be any semi-prime ring and $f$ be a generalized inner derivation of $A$. Then $h_{a}$ must neccessarily be a derivation. 
ISSN No:-2456-2165

\section{Proof. Now}

$f(x y z)=f(x) y z+x h_{a}(y z)$.

Also

$$
\begin{aligned}
f(x y z) & =f(x y) z+x y h_{a}(z) \\
& =\left(f(x) y+x h_{a}(y)\right) z+x y h_{a}(z)
\end{aligned}
$$$$
=f(x) y z+x h_{a}(y) z+x y h_{a}(z)(2)
$$

From (1) and (2), we get

$$
\begin{aligned}
& x\left(h_{a}(y z)-h_{a}(y) z-y h_{a}(z)\right)=0 \\
& \text { Now } f(x(y+z)) \quad=f(x)(y+z)+x h_{a}(y+z) \\
& =f(x) y+f(x) z+x h_{a}(y+(4)
\end{aligned}
$$

$z)$.

Also

$$
\begin{aligned}
f(x(y+z)) & =\quad f(x y+y z) \\
& =\quad f(x y)+f(x z) \\
& =f(x) y+x h_{a}(y)+f(x) z+(5)
\end{aligned}
$$

$x h_{a}(z)$

From (4) and (5), we have

$x\left(h_{a}(y+z)-h_{a}(y)-h_{a}(z)\right)=0$

Since $A$ is semi prime ring and if $A a=0$ Then $a=0, a \in A$. $\Rightarrow$ From (3) and (6), we have

$$
\begin{array}{r}
h_{a}(y z)-h_{a}(y) z-y h_{a}(z) \\
h_{a}(y+z)-h_{a}(y)-h_{a}(z) \\
\Rightarrow h(y z)=h_{a}(y) z+y h_{a}(z) h_{a}(y+z)=h_{a}(y)+h_{a}(z)
\end{array}
$$

Hence $h_{a}$ is a derivation. Hence proved.

Corollary 2.3 $f(x y)=f(x) y+x h_{a}(y)$ where $h_{a}$ is a derivation replacing $h_{a}$ by $d$, we get Havala [2] def. P.1147 of Generalized derivation

Corollary 2.4 Havala [2] result is also proved. "Let $A$ be any semi prime ring. Then $f(x y z)=f(x) y x+x y d(x)+$ $x d(y) x \forall x, y \in A$

where $f$ is generalized derivation of $A$."

Corollary 2.5 Replacing $f$ by $d$ in Corollary 2.4, we get Lemma 3.1

P.1106 of Herstein [3]

$d(x y z)=d(x) y x+x d(y) x+x y d(x)$

$C$. In this section, we take $A$ be any 2 torsion free semiprime ring.

Definition 3.1. Let $A$ be any ring which is 2 torsion free and also semi prime. We define

$x \circ y=x y+y x \forall x, y \in A$
Theorem 3.2 Let $A$ be any ring which is 2 torsion free and also semi prime with Identity 1 . Then $\exists$ no generalized inner derivation $f: A \rightarrow A$ such that

$$
f(x \circ y) \quad=x \circ y \forall x, y \in A
$$

or $f(x \circ y)+x \circ y=$

0 .

Proof. If possible, let $\exists$ a generalized derivation $f: A \rightarrow A$ s.t $f(x \circ y)=x \circ y$ or $f(x \circ y)+x \circ y=\quad 0 \forall x, y \in A$.

Now

or

$$
\begin{aligned}
f(x \circ y) & =x \circ y \\
f(x \circ y)+x \circ y & =0
\end{aligned}
$$

Putting $y=1$

$\Rightarrow f(x \circ 1)=x \circ 1$

or $f(x \circ 1)+x \circ 1=0$

$\Rightarrow f(x+x)=x+x$

or $f(x+x)+x+x=0$

$\Rightarrow f(2 x)=2 x$

or $f(2 x)+2 x=0$

$\Rightarrow f(2 x)=2 x$

or $f(2 x)=-2 x$

$\Rightarrow f(2 x)= \pm 2 x$

$\forall x \in A \& A$ is 2 -Torsion free.

$$
\begin{aligned}
& \text { Now } x y+y x=\quad x \circ y= \pm f(x \circ y) \\
& = \pm f(x y+y x) \\
& =\quad(-(x y+y x)) \quad(\because f(x \circ y)=-(x \circ y)) x y+y x \\
& =\mp(x y+y x) \\
& \Rightarrow 2(x y+y x) \quad=0 \\
& \Rightarrow x \circ y=0
\end{aligned}
$$$$
=0
$$

$=0$ Since $A$ is 2-Torsion free $\Rightarrow x^{2}=0$

Now

$$
\begin{aligned}
& \begin{array}{ll}
x \circ y & =0
\end{array} \\
& \Rightarrow x \circ(x+1)=\quad 0 \quad \text { (Taking } y=x+1) \\
& \Rightarrow 2 x \quad=\quad 0 \\
& \Rightarrow x \quad=\quad 0 \forall x \in A
\end{aligned}
$$

which is a contradiction ( $\because$ Identity is 1 ).Hence our supposition is wrong.

So, $\exists$ no generalized inner derivation $f$ satisfying

or

$$
f(x \circ y)=x \circ y
$$

$$
f(x \circ y)+x \circ y \quad=0 \quad \forall x, y \in A
$$

Hence proved.

$D$. In this section, we take $A$ be any ring with unity and we consider non-zero Ideal of $A$

Lemma 4.1 Let $K 6=\{0\}$ be an Ideal of A with unity and $f$ be a 
G.I.D of $A$. Then $\forall x, y \in K$ satisfying $x y+f(x y)=y x+f(y x)$ $(1+f(1))[x, y]+[a,[x, y]]=0$

Proof.

$x y+f(x y)=y x+f(y x)$

$\Rightarrow x y+f(x) y+x h_{a}(y)=y x+f(y) x+y h_{a}(x)$

$\Rightarrow x y+\left(f(1) x+1 h_{a}(x)\right) y+x h_{a}(y)=y x+\left(f(1) y+1 h_{a}(y)\right) x+$

$y h_{a}(x)$

$\Rightarrow x y+f(1) x y+a x y-x y a=y x+f(1) y x+a y x-y x a$

$\Rightarrow(x y-y x)+f(1) x y-f(1) y x+a x y-x y a-a y x+y x a=0$

$\Rightarrow[x, y]+f(1)[x, y]+a(x y-y x)-(x y-y x) a=0$

$\Rightarrow[x, y]+f(1)[x, y]+a[x, y]-[x, y] a=0$

$\Rightarrow[x, y]+f(1)[x, y]+[a,[x, y]]=0$

$\Rightarrow(1+f(1))[x, y]+[a,[x, y]]=0$ Hence proved.

\section{CONCLUSION}

We showed that for any Ring A which is semi prime , $h_{a}$ is a derivation. We also proved Havala [2] def. p.1147, Herstein [3] Lemma 3.1 p. 1106 as corollaries of our results.

\section{REFERENCES}

[1]. Matej Bresar, On the distance of the composition of two derivations to Generalized derivations, Glasgow Math. J. 33 (1991), Page 89-93.

[2]. B. Havala, Generalized Derivations in rings, Communication in Algebra, 26 [4], 1998, Page 11471166.

[3]. I. N. Herstein, Jordan Derivations of Prime rings, Proc. Amer. Math. Soc.,8 (1957), 1104-1110.

[4]. M. Hongan, A note on semi-prime rings with derivation, International J. Math. and Math. Sci. Vol. 20 No. 2 (1997), Page 413-415. 